\title{
Diffusive properties of colloidal charged particles in a quasi-one-dimensional
}

\section{confinement}

\author{
Propriedades difusivas de partículas coloidais carregadas em um confinamento quasi- \\ unidimensional \\ Propiedades difusivas de partículas coloidales cargadas en confinamiento cuasi-unidimensional
}

Received: 09/14/2021 | Reviewed: 09/19/2021 | Accept: 09/23/2021| Published: 09/25/2021

Levi Rodrigues Leite

ORCID: https://orcid.org/0000-0003-3986-3551 Universidade da Integração Internacional da Lusofonia Afro-Brasileira, Brasil

E-mail: levi@unilab.edu.br

Jorge Luiz Bezerra de Araújo

ORCID: https://orcid.org/0000-0001-5101-6246

Universidade de Fortaleza, Brasil

E-mail: jorgearaujo@unifor.br

Leandro Jader Pitombeira Xavier

ORCID: https://orcid.org/0000-0003-0458-9994

Instituto Federal do Ceará, Brasil

E-mail: leandro.jader@ifce.edu.br

Vagner Henrique Loiola Bessa

ORCID: https://orcid.org/0000-0002-7584-262X

Instituto Federal do Ceará, Brasil

E-mail: vagner.bessa@ifce.edu.br

João Cláudio Nunes Carvalho

ORCID: https://orcid.org/0000-0001-8619-0869

Instituto Federal do Ceará, Brasil

E-mail: joao.carvalho@ifce.edu.br

Diego de Lucena Camarão

ORCID: https://orcid.org/0000-0002-3497-4968

Universidade de Fortaleza, Brasil

E-mail: diego@ fisica.ufc.br

\begin{abstract}
Diffusive properties of colloidal crystals in a quasi-one-dimensional channel are studied using numerical simulations. In order to study the influence of the attractive interaction between particles, it was introduced as an artificial dimensionless parameter $\beta$ in the attractive term of the interaction potential. Changing the value of $\beta$, we can tune the effect of attraction between particles. We show that charged particles can change their mobility and the diffusion exponent of a one-chain like system. Variation on exponent diffusion can be induced by tuning the attractive part of interaction potential, making possible the existence of diffusive regimes between single-file diffusion (SFD) and normal diffusion, without changing confinement strength. System stoichiometry was changed, imposing particles in different arrangements in small clusters, which varies the diffusive behaviour. If stoichiometry is different from 1:1, it is possible to have particles with equal charges but with different mobilities. Another important observation is that mean-square displacement (MSD) for different charges is different for different values.
\end{abstract}

Keywords: Diffusion; Colloids; Single-file.

\section{Resumo}

Propriedades difusivas de cristais coloidais em um canal quase unidimensional são estudadas usando simulações numéricas. Para estudar a influência da interação atrativa entre partículas, foi introduzido como um parâmetro adimensional artificial $\beta$ no termo atrativo do potencial de interação. Alterando o valor de $\beta$, podemos ajustar o efeito da atração entre as partículas. Mostramos que partículas carregadas podem mudar sua mobilidade e o expoente de difusão de um sistema semelhante a uma cadeia. A variação na difusão do expoente pode ser induzida pelo ajuste da parte atrativa do potencial de interação, possibilitando a existência de regimes difusivos entre a difusão single-file (SFD) e a difusão normal, sem alterar a força de confinamento. A estequiometria do sistema foi alterada, impondo partículas em diferentes arranjos em pequenos aglomerados, o que varia o comportamento difusivo. Se a estequiometria for diferente de 1:1, é possível ter partículas com cargas iguais, mas com mobilidades diferentes. Outra observação importante é que o desvio quadrático médio (MSD) para cargas diferentes é diferente para valores diferentes.

Palavras-chave: Difusão; Colóides; Single-file. 


\begin{abstract}
Resumen
Las propiedades de difusión de los cristales coloidales en un canal cuasi unidimensional se estudian mediante simulaciones numéricas. Para estudiar la influencia de la interacción atractiva entre partículas, se introdujo como un parámetro adimensional artificial $\beta$ en el término atractivo del potencial de interacción. Cambiando el valor de $\beta$, podemos sintonizar el efecto de atracción entre partículas. Mostramos que las partículas cargadas pueden cambiar su movilidad y el exponente de difusión de un sistema similar a una cadena. La variación en la difusión del exponente se puede inducir sintonizando la parte atractiva del potencial de interacción, lo que hace posible la existencia de regímenes de difusión entre la difusión en una sola fila (SFD) y la difusión normal, sin cambiar la fuerza del confinamiento. Se modificó la estequiometría del sistema, imponiendo partículas en diferentes arreglos en pequeños racimos, lo que varía el comportamiento difusivo. Si la estequiometría es diferente de 1:1, es posible tener partículas con cargas iguales pero con diferentes movilidades. Otra observación importante es que el desplazamiento cuadrático medio (MSD) para diferentes cargas es diferente para diferentes valores.
\end{abstract}

Palabras clave: Difusión; Coloides; Single-file.

\title{
1. Introduction
}

Diffusion can be defined as the movement of particles under influence of a concentration gradient. When particles are moving in space with reduced dimensionality, or under the influence of an external confinement, interesting diffusion behaviours can emerge. A particular case of confined geometry happens when particles are restricted to move along a line in a channel so narrow that particles cannot cross each other, so the sequence of particle labels does not change in time. This impossibility of particles crossing induces a collective motion of particles in the same direction. In this situation, anomalous diffusion can be observed (Harris, 1965) such that the mean-square displacement,

$$
\left\langle\Delta x^{2}(t)\right\rangle \propto \sqrt{t}
$$

This result is in contrast with normal diffusion, where the mean-square displacement grows proportional to time. This phenomenon is called Single-file diffusion (SFD), and it has been studied in different contexts in monodisperse systems. M. Kollmann (Kollmann, 2003) proposed an analytical approach in which SFD regimes appear independently of the nature of interactions for homogeneous systems in the fluid state. Guthmann (Coupier et al, 2006) and Nelissen et al (Nelissen et al, 2007) showed that interaction potential can lead to different subdiffusive behavior, with diffusive exponent lower than the square root of time. From now on different studies of interaction potential appear: dipolar, Yukawa, periodic substrate, etc. (Lucena et al, 2012; Carvalho et al, 2012; Carvalho et al, 2011) for different damping values (Delfau et al, 2011) and different connement intensities and traps in a monodisperse system of interacting particles (Lucena et al, 2012). Single-file diffusion are also present in nature in many different situations such as diffusion experiments with molecules of zeolite (Meier, 1992), in colloids (Konig, 2005; Wei, 2000), charged macroscopic beads (Coupier et al, 2006) and water through molecular-sized channels in membranes (Hernandez, 1992; Morais-Cabral et al, 2001; Doyle et al, 1998) and for magnetic particles and dipoles (Lucena et al, 2014; Galvan-Moya et al, 2014). In SFD, particles are correlated because particle movement induces other particles to move together.

When potential interactions are a long-ranged attraction, colloidal particles tend to aggregate. M. E. Leunissen et al showed that the electrostatic interaction between oppositely charged particles can be tuned such that large ionic colloidal crystals can form stable structures. It was found that in contrast to atomic systems, the stoichiometry of colloidal crystals is not dictated by charge neutrality; this allows the existence of a remarkable diversity of new binary structures (Leunissen et al, 2005). Advances in experimental and numerical techniques allowed a considerable improvement to create and study new cristalyne structures. An interesting property of binary crystals is the self-organization in a two-dimensional (2D) binary colloidal system. Recently, a rich variety of binary superlattices were obtained experimentally (Leunissen et al, 2005; Shevchenko, 2006), when for different stoichiometries, new crystalline superlattices appeared. In this work we study diffusive 
properties of colloidal crystals under a parabolic confinement, for three different stoichiometries.

\section{Methodology}

\section{A. Colloidal crystals}

Our methodology consists of creating a program in FORTRAN language which simulates colloids interacting one another in a box with periodic boundary conditions, to simulate an infinite system. The numerical technique applied is called Molecular Dynamics. Molecular dynamics simulations can be divided into the following steps: (1) put particles in a simulation box; (2) calculate the force that particles exerts one each other; (3) integrate equations of motion (in this work we used Euler method) and (4) calculation of the properties in which we are interested (Frenkel \& Smit, 2002).

Our model of colloidal crystal consists of $\mathrm{N}+=50$ particles with positive charge $\mathrm{q}+$ and $\mathrm{N}-=50$ particles with negative charge q-. The system as a whole is electrically neutral, i.e., $\mathrm{N}+\mathrm{q}++\mathrm{N}-\mathrm{q}-=0$. The movement of the $\mathrm{N}=\mathrm{N}++\mathrm{N}-$ particles in the xy-plane is restricted along the y direction by a one-dimensional parabolic potential. Since the parabolic confinement allows particles to occupy regions of two-dimensional space with y different from 0 the present system is usually called quasi-one-dimensional (q1D). The equation of motion which describe the movement of the $\mathrm{i}$-th colloid is given by the Langevin equation

$$
m \dot{\vec{v}}_{i}=-\gamma m \vec{v}_{i}+\vec{f}_{i}+\vec{f}_{i}^{e x t}+\vec{f}_{i}^{T}(t)
$$

where $\mathrm{m}$ is the mass of the each particle, $\gamma$ is the damping constant, $\vec{f}_{i}=-\sum_{i \neq i} \vec{\nabla} V_{i j}, \vec{f}_{i}^{\text {ext }}=-\vec{\nabla} V_{i}^{\text {ext }}$ and $\vec{f}_{i}^{T}(t)_{\text {is a random Gaussian force with known properties }}$

$$
\begin{aligned}
\left\langle f_{i}^{T}(t)\right\rangle & =0 \\
\left\langle f_{i m}^{T}(t) f_{j n}^{T}\left(t^{\prime}\right)\right\rangle & =2 \gamma m k_{B} T \delta_{i j} \delta_{m n} \delta\left(t-t^{\prime}\right),
\end{aligned}
$$

where $k_{B}$ is the Boltzmann constant and $\mathrm{T}$ is the absolute temperature.

The interparticle interaction pair potential is given by

$$
V_{i j}=\frac{q_{i} q_{j}}{4 \pi \epsilon} \frac{\exp \left(-r_{i j} / \lambda\right)}{r_{i j}}+B\left(\frac{\sigma}{r_{i j}}\right)^{12},
$$

where $\epsilon$ is the dielectric constant of the medium the particles are moving in, $\lambda$ is the Debye screening length, qi is the

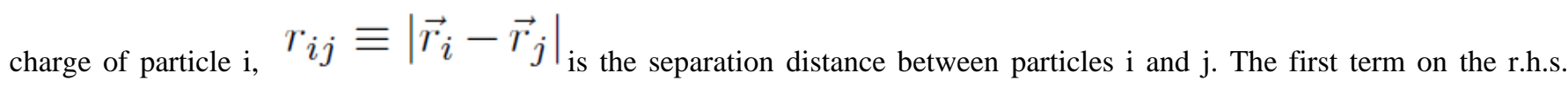
(right-hand side) of Eq. (4) is the Debye-Hückel (or Yukawa) potential. The second term in the same equation is a soft-core repulsion. $\sigma$ defines the distance between the centers of the particles $\mathrm{i}$ and $\mathrm{j}$ when those particles are in contact and $\mathrm{B}$ is an energy parameter in order to prevent particles from coalescing into a single point. The soft-core repulsion is introduced to avoid particle-particle overlap, since the product qi $x$ qj can be negative depending on the modulus of the charge of each particle.

The external one-dimensional parabolic potential confinement is modelled by an harmonic trap 


$$
V_{i}^{e x t}=\frac{1}{2} m \omega^{2} y_{i}^{2},
$$

where $\omega$ is the strength of the parabolic external confinement and yi is the y coordinate of the ith particle.

\section{B. Dimensionless units and numerical integration}

We consider $\mathrm{q}+=+\mathrm{Zq}$ and $\mathrm{q}-=-\mathrm{q}$, where $\mathrm{q}+$ and $\mathrm{q}-$ is the charge of positive and negative particles, respectively. We choose $\mathrm{q}$ as unit charge, and $\mathrm{Z}=\mathrm{q}+\mathrm{q}$ - is therefore the stoichiometry. Considering that particles have a constant charge surface density $\quad \alpha=q_{ \pm} / 4 \pi R_{ \pm}^{2}$, and that $a$ is the radius of the negative particle -q, we have $\sigma_{++}=2 \sqrt{Z} a, \sigma_{--}=2 a$ and $\sigma_{+-}=(\sqrt{Z}+1) a \cdot \sigma_{++}$is the sum of positive particles radius, $\sigma_{-}-$is the sum of negative particles radius and $\sigma_{+-}$is the sum of a positive with a negative particle radius (see Fig.1).

Figure 1 - Schematic representation of model system. Positive particles have radius R+ and negative particles have radius R-.

The sum of particles radius are represented by letter, where $\sigma_{++}=R_{+}+R_{+}, \sigma_{--}=R_{-}+R_{-}$and

$$
\sigma_{+-}=R_{+}+R_{-} \text {. }
$$
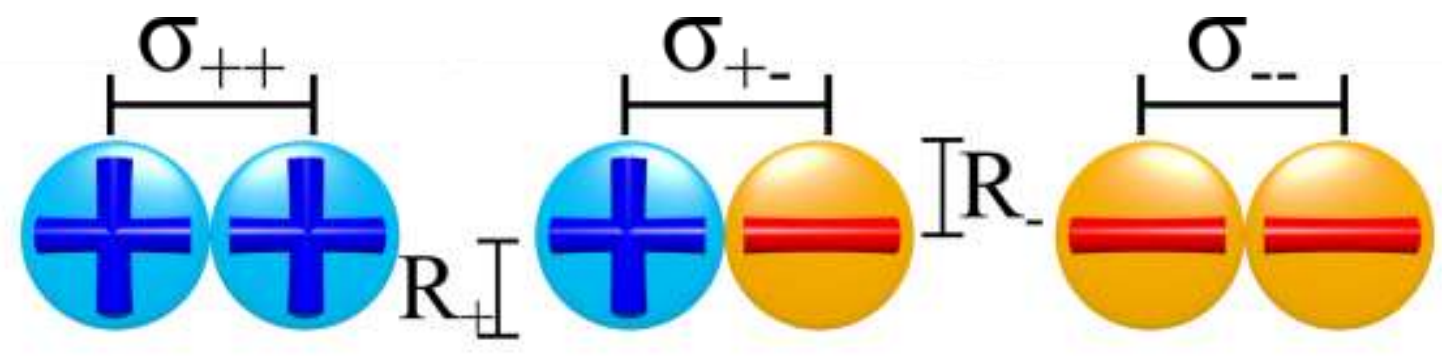

Source: Image created by the author.

By choosing the unit of distance as a and the unit of energy as $k_{B} T$, the dimensionless (tilde) interparticle interaction potential is written as

$$
\begin{aligned}
& \tilde{V}_{i j}=\tilde{\Gamma} \tilde{Z}^{2} \frac{\exp \left(-\tilde{\kappa} \tilde{r}_{i j}\right)}{\tilde{r}_{i j}}+\tilde{B}\left(\frac{\sigma_{+}+}{\tilde{r}_{i j}}\right)^{12}+ \\
& \tilde{\Gamma} \frac{\exp \left(-\tilde{\kappa} \tilde{r}_{i j}\right)}{\tilde{r}_{i j}}+\tilde{B}\left(\frac{\sigma_{-}}{\tilde{r}_{i j}}\right)^{12}- \\
& \beta \tilde{\Gamma} \tilde{Z} \frac{\exp \left(-\tilde{\kappa} \tilde{r}_{i j}\right)}{\tilde{r}_{i j}}+\tilde{B}\left(\frac{\sigma_{+}}{\tilde{r}_{i j}}\right)^{12},
\end{aligned}
$$


where $\tilde{\Gamma}=\frac{q^{2}}{4 \pi \epsilon k_{B} T a}, \tilde{B}=\frac{B}{k_{B} T}$ and $\tilde{\kappa}=\frac{a}{\lambda}$. The dimensionless (tilde) external one-dimensional parabolic potential confinement is written as

$$
\tilde{V}_{i}^{e x t}=\frac{1}{2} \tilde{\chi}\left(\tilde{y}_{i}\right)^{2}
$$

where $\tilde{\chi}=\frac{m \omega^{2} a^{2}}{k_{B} T}$ is the dimensionless parameter which regulates the external confinement potential.

In order to study the influence of the attractive interaction between particles, note that it was introduced as an artificial dimensionless parameter $\beta$ in the last term of equation (6). Changing the value of $\beta$, we can tune the effect of attraction between particles.

Furthermore, the unit of time is given by $t_{0}=a \sqrt{\left(m / k_{B} T\right)}$. In our simulations, we use $\tilde{\Gamma}=100, \tilde{B}=1, \tilde{\kappa}=1, \tilde{\gamma}=\gamma t_{0}=5, \tilde{\chi}=200, \tilde{q}_{+}=+1_{\text {and }} \tilde{q}_{-}=-1$ in all cases, except when specified otherwise. In all simulations, density was defined as the ratio between number of particles and the box size, and its value is always equal to 0.2. From this point on, we drop the tilde notation and all quantities are dimensionless.

The numerical integration of the dimensionless form of Eq. (2) is given by the Gillespie (Gillespie, 1996(54); Gillespie, 1996(64)) algorithm

$$
\begin{aligned}
\vec{v}_{i}(t+\Delta t) & \simeq \vec{v}_{i}(t)-\gamma \vec{v}_{i}(t) \Delta t+\left(\vec{f}_{i} / m\right) \Delta t+ \\
& +\left(\vec{f}_{i}^{e x t} / m\right) \Delta t+\sqrt{(2 \gamma T \Delta t) / m} \vec{\eta}_{i}
\end{aligned}
$$

to update the particles' velocities, where $\Delta t=10^{-4}$ is the dimensionless time step and $\vec{\eta}_{i}$ is the dimensionless form of the random Gaussian force (Eq. (3)). The position of the particle is then updated using the formula

$$
\vec{r}_{i}(t+\Delta t)=\vec{r}_{i}(t)+\vec{v}_{i}(t) \Delta t
$$

\section{Results and Discussion}

\section{A. Mean-Square Displacement}

In this section, we present our results about particles' diffusive regimes. In order to study it, we calculated MSD as follows:

$$
\left\langle\Delta x^{2}(t)\right\rangle=\left\langle\frac{1}{N} \sum_{i=1}^{N}\left[x_{i}(t+\Delta t)-x_{i}(t)\right]^{2}\right\rangle_{\Delta t}
$$


where $\mathrm{N}$ is the number of particles, \langle\rangle$\Delta t$ represents a time average over the time interval $\Delta t$, and $\mathrm{x}$ is the particle position.

In general it is possible to distinguish three different regimes: (i) a short-time regime (STR), where particles diffuse ballistically (i.e., $\left\langle\Delta x^{2}(t)\right\rangle \propto t^{2}$ ) (ii) an intermediate time regime (ITR) where particles start to interact and change their mobilities and (iii) a long-time regime (LTR), where particles diffuse as a whole body. In the following subsections these three different steps are explained and its relationship with charges in different stoichiometries.

\section{B. 1:1 Stoichiometry}

For stoichiometry $1: 1$, we have N/2 of positive particles and N/2 of negative particles. We can observe that these particles have the same size. MSD calculations are shown in Figure 2.

Figure 2 - MSD calculation for five different $\beta$ values (from 0 to 1.0, with variation of $\beta$ by 0.25 ). Dashed line illustrates normal diffusion regime and dotted lines illustrates single-file diffusion regime. Dashed-dotted line shows that for $\beta=0.75$, diffusion exponent is 0.7 , and for $\beta=1.00$ diffusion exponent is 0.6 .

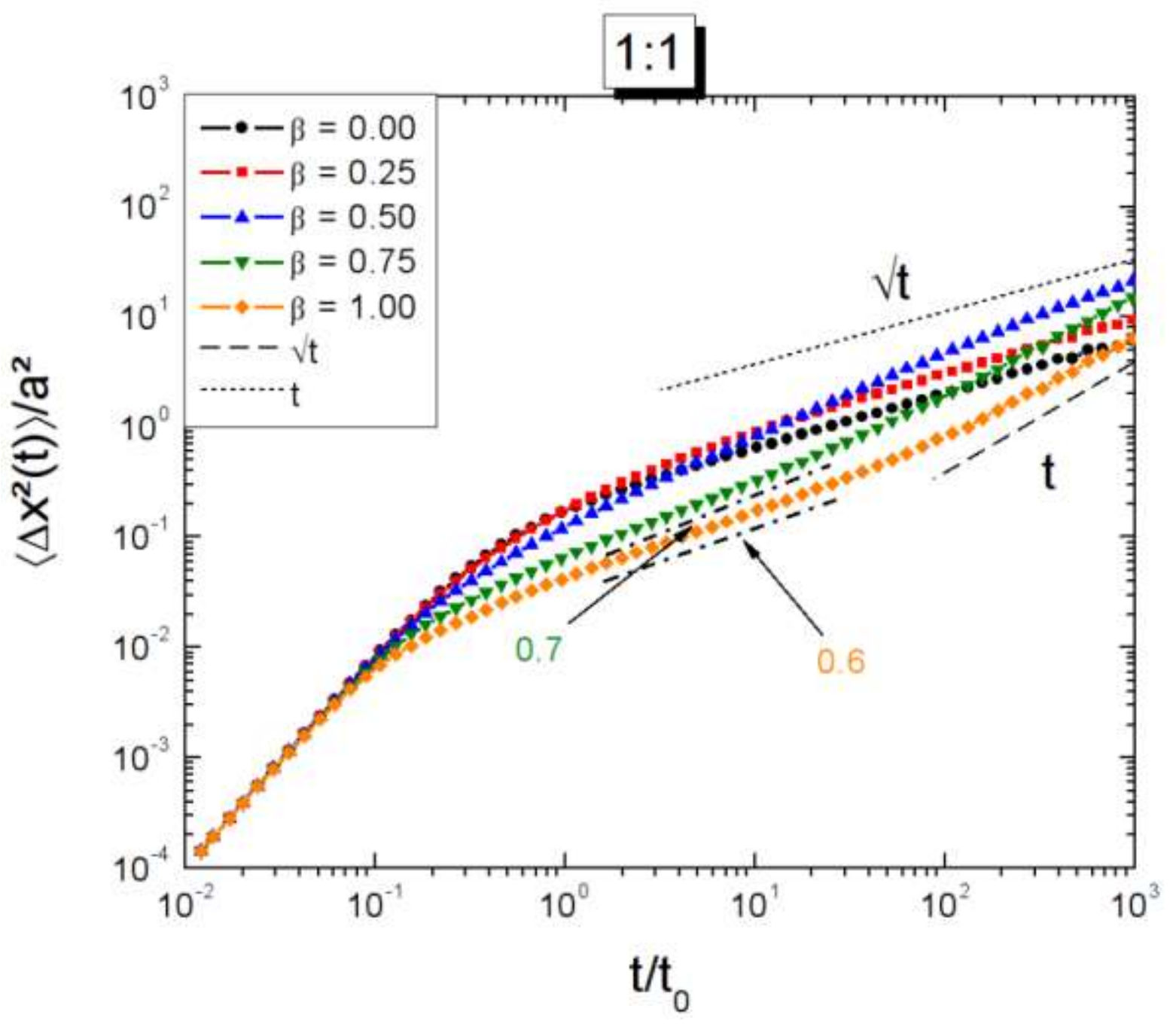

Source: Image created by the author.

For STR, we always obtain a ballistic regime, for all values of $\beta$. But if we look at ITR we have different situations 
for different $\beta$ values. For $\beta=0.0,0.25$ and 0.50 , we observe that particles reach the SFD regime. But when $\beta=0.75$, we have that diffusion exponent is equal to 0.7 , and if $\beta=1.0$ (repulsion and attraction in equal intensities) we have a diffusion exponent equal to 0.6. This result shows that in conned channel-like geometries, it is possible to obtain exponents between 1.0 and 0.5 without changing the connement intensity. Another observation for $\beta=0.75$ and 1.0 is that in LTR particles approaches exponent 1.0, behaving as only one body. This happens because when $\beta$ is between $0.75-1.0$ the final structure is a set of clusters that emerges because attractive interaction is much stronger than repulsive interaction, as shown in Figure 3. Temperature is high enough such that these clusters can break and get linked in another small cluster.

Figure 3 - Equilibrium configuration for a system with 1:1 stoichiometry. Blue "+" circles are positive particles and red "-" circles are negative particles.

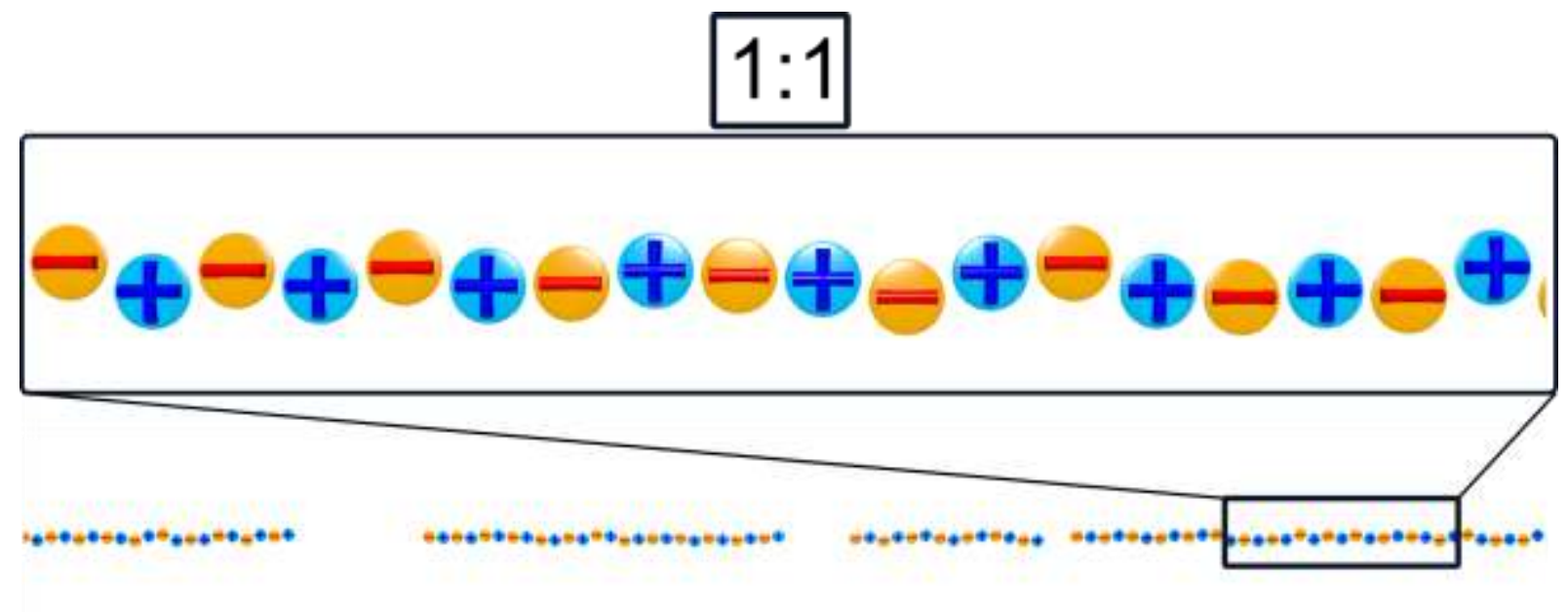

Source: Image created by the author.

In order to explain why strong attractive interaction leads to diffusion exponents higher than 0.5, Figure 3 should be analyzed. We see that the final configuration is a set of clusters which change particles one at a time, with empty spaces between clusters along the unconfined direction. When particles go from one cluster to another, this movement is induced by attractive interaction which grows as the particles approach one another, and stops when soft-core repulsion starts to act. This accelerated movement induced by attractive force is more relevant on hierarchy of forces than repulsive interaction so that implies a diffusion exponent with higher value than 0.5 . It is also important to observe that when $\beta=0.75$, temperature also breaks more easily when particles agglomerate than when $\beta=1.0$, which explains that for $\beta=0.75$ exponent diffusion is higher than for $\beta=1.0$.

To understand the increase of exponent diffusion, we calculate the mean-square displacement of each jth particle $[\mathrm{Wj}(\mathrm{t})]$ by equation

$$
W_{j}(t)=\left\langle\left[x_{j}(\tau+\delta t)-x_{j}(\tau)\right]^{2}\right\rangle_{\tau}
$$

where $\mathrm{j}=1, \ldots, \mathrm{N}$ represents each individual particle and $\langle.\rangle_{\tau}$ is an average over different time origins during simulation. In Figure 4 we see that some particles have diffusion exponents equal to 0.7 (higher value) and other particles equal to 0.55 (lower value). This means that some particles tend to stay more time moving themselves from one cluster to another (higher exponent value), while other particles tend to stay at the center of the cluster (lower exponent value), inducing the mean-value of diffusion exponent slightly larger than 0.5 . 
Figure 4 - Mean-square displacement of the system (white circle, W(t)) and mean-square displacement of individual particles (black circles, $\mathrm{Wj}(\mathrm{t})$ as a function of time $\mathrm{t}$ for $\beta=1.0$ and stoichiometry $1: 1$. )

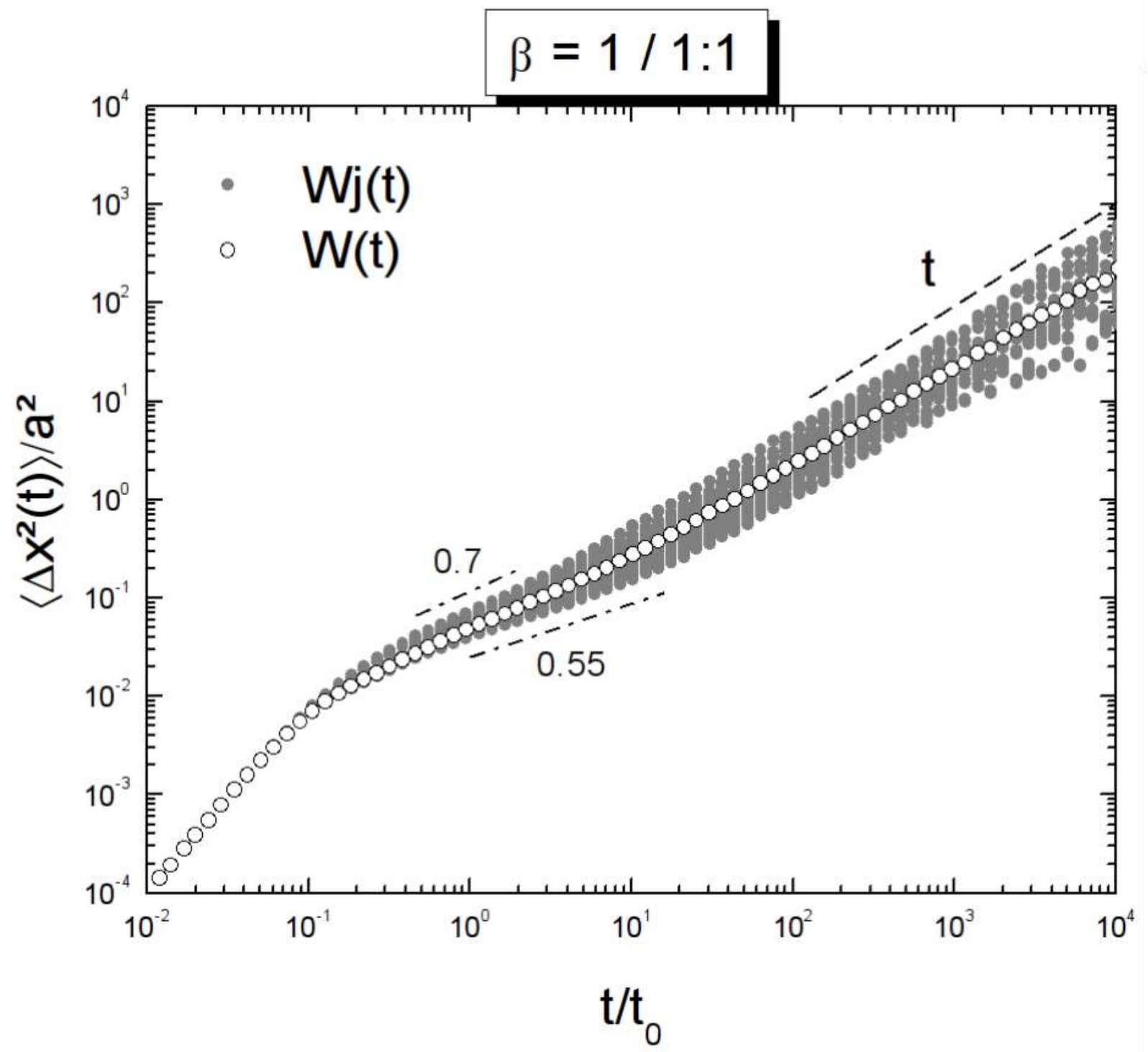

Source: Image created by the author.

A particle with positive charge has negative nearest neighbors and vice-versa, so attractive interaction becomes more relevant and the big cluster appears. Then when it is great enough to make attraction greater than repulsion, the way particles feel one another is different, which explains the different exponents for ITR.

Another important point is that time transition between STR and ITR decreases as attractive interaction increases. This happens because neighbor particles are always of opposite charge, so greater attractivity implies more fast interactions and more fast diffusion regime transition.

Plotting positive and negative charges separately shows that they diffuse in equal manner. This is expected because particles feel the same forces by its neighbours, in both structures (free particles or cluster structure), as shown in Figure 5. 
Figure 5 - MSD for a) $\beta=0$ and b) $\beta=1: 0$. Black solid line is the MSD curve for positive particles and the red circles are the MSD curve for negative particles. Curves are almost exactly the same.

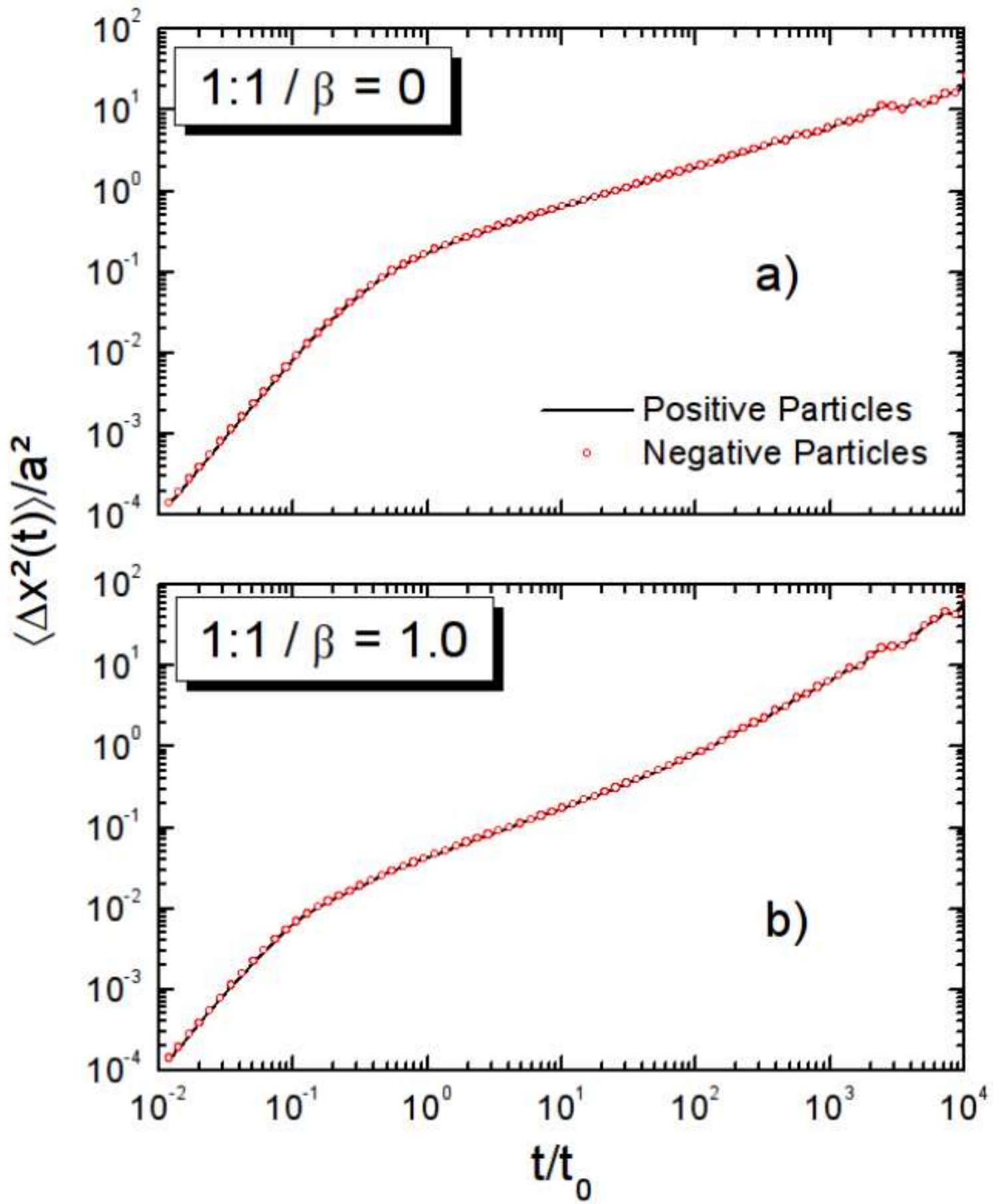

Source: Image created by the author.

\section{2:1 Stoichiometry}

For stoichiometry $2: 1$, we have N/3 of positive particles and $2 \mathrm{~N} / 3$ of negative particles. In this case positive particles size is $\sqrt{2} a$, where $a$ is the radius of negative particles. MSD calculations are shown in Fig. 6. 
Figure 6 - MSD curves for different values of $\beta$. Dashed line illustrates normal diffusion regime and dotted lines illustrates single-file diffusion regime.

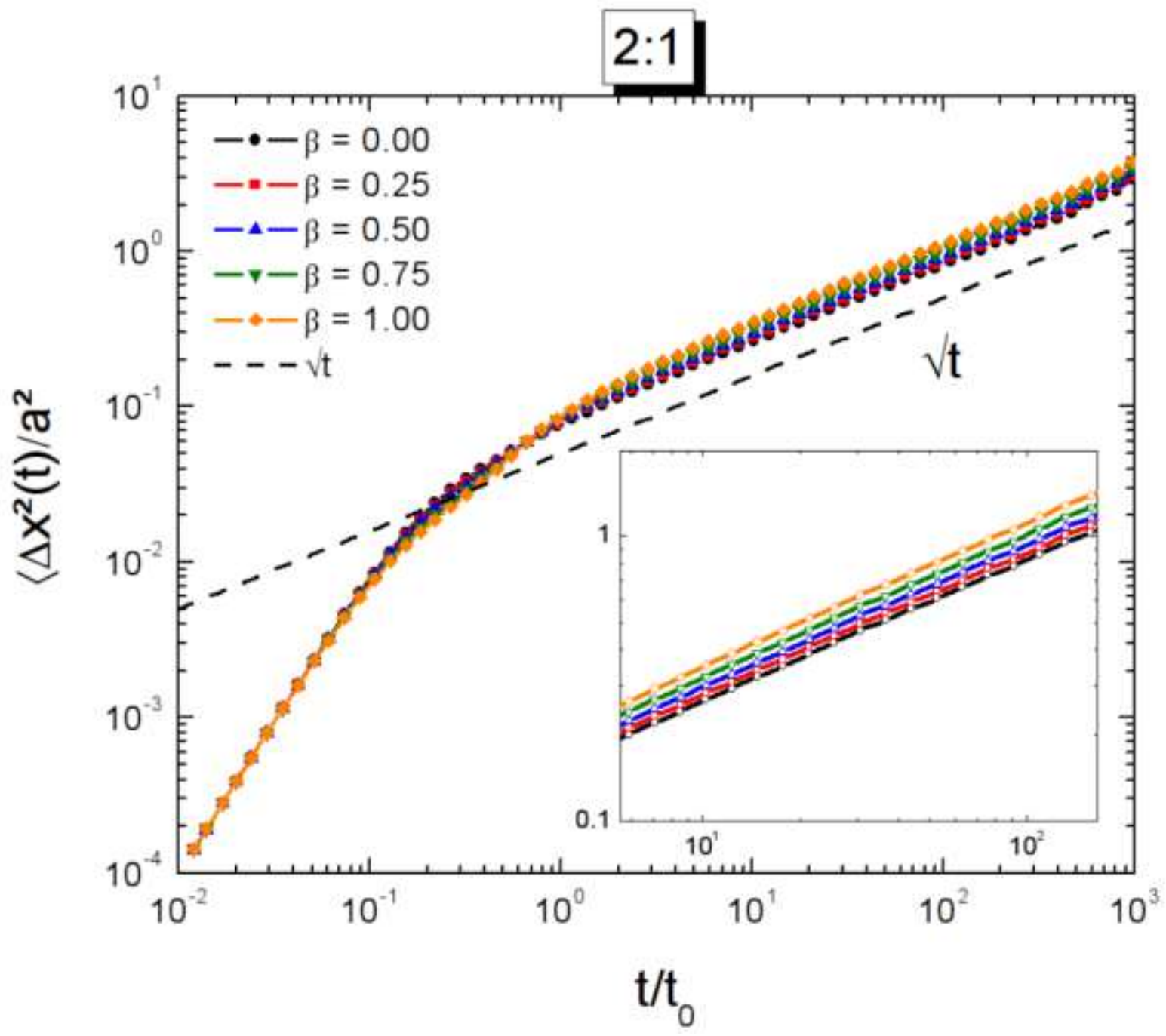

Source: Image created by the author.

For STR, ballistic regime is always attained. When ITR is analyzed, we can observe that SFD is always reached. Why do attractive interactions not lead to diffusion exponents between 0.5 and 1.0 ? In order to understand this point, final structures are sketched in Figure 7. 
Figure 7 - Equilibrium configuration for system with 2:1 stoichiometry. Blue "+" circles are positive particles and red "-" circles are negative particles.

$$
\text { 2:1 }
$$

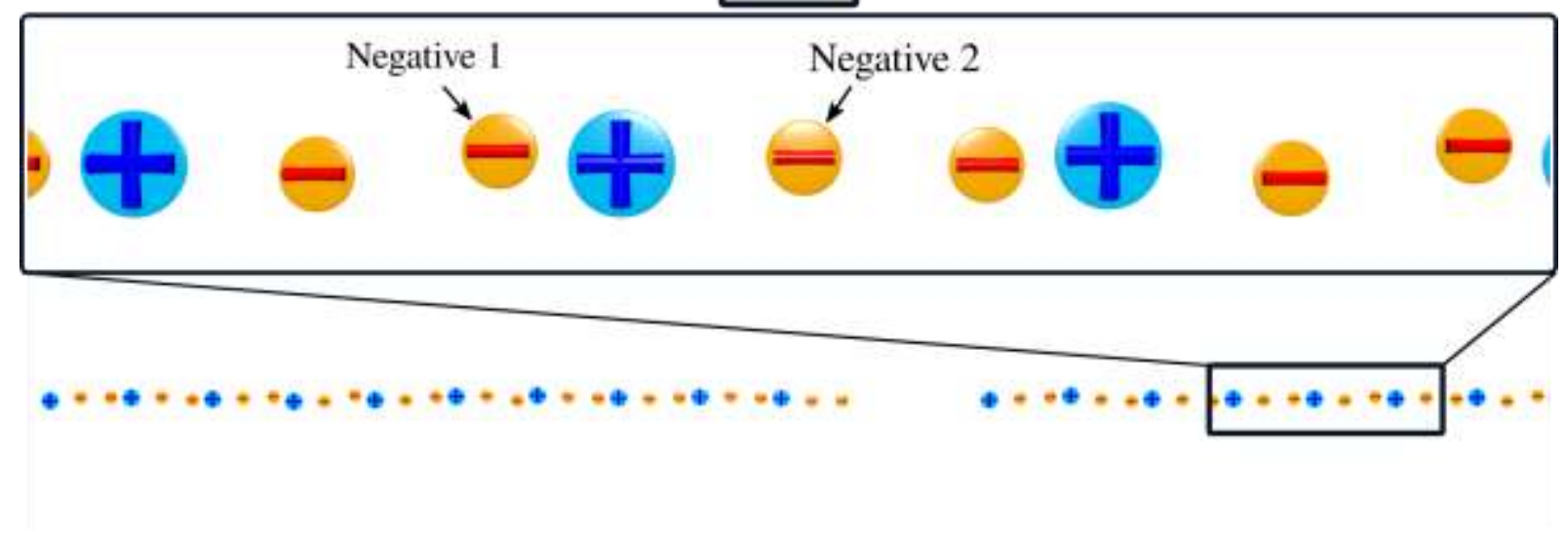

Source: Image created by the author.

When equilibrium is reached, positive particles attract negative particles to their surroundings, creating molecules with trimmerlike form. These negative particles create a screened trimmer. The consequence is that trimmers behave as single molecules with repulsive interactions more relevant than attractive one. This is the reason that trimmers diffuse always in SFD. Another fact is that when it increases its value, particle mobility also increases. This happens because when attractive force increases, the positive particle attracts negative particles in a more intense manner, which means that trimmer particles are closer to one another, then trimmers get a lower size. With a lower size, trimmers have more space to diffuse, increasing its mobility. Another consequence is that trimmers need more time to "feel" one another, which explains why time transition from STR to ITR raises together with values.

For 2:1 stoichiometry, negative and positive particles diffuse with a tight difference, as shown on Figure 8. 
Figure 8 - MSD for a) $\beta=0$ and b) $\beta=1: 0$. Black solid line is MSD curve for positive particles, the red solid line is the MSD for Negative 1 particles and blue circles are MSD curve for Negative 2 particles.

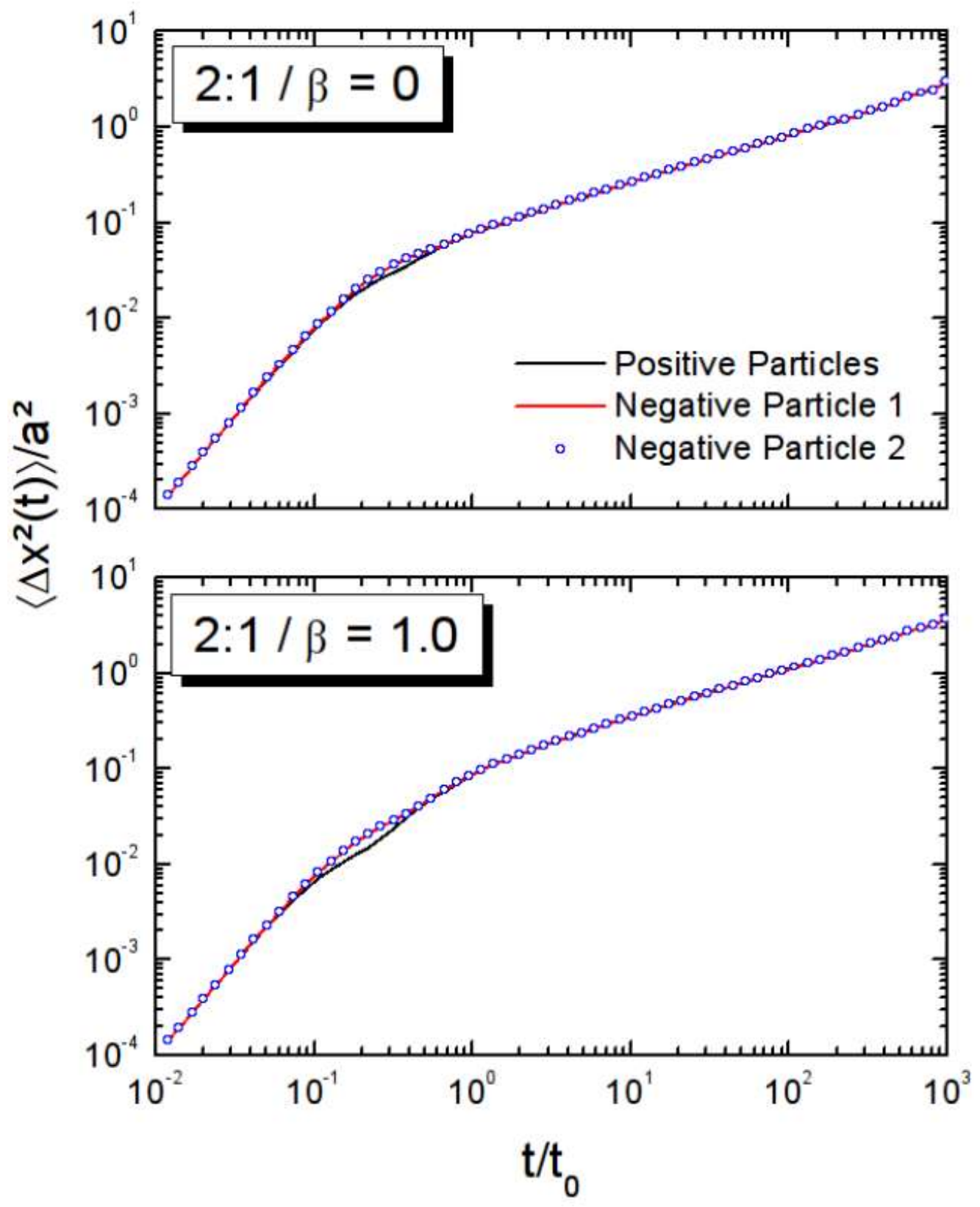

Source: Image created by the author.

We see that in transition from STR to ITR, positive particles have a smaller diffusion coecient. Positive particles in 2:1 stoichiometry exerts a stronger attraction on negative particles on both sides, creating trimmer molecules. This implies that negative particles go in the positive particles direction, which leads to lower diffusion coefficient by positive particles in STRITR transition.

\section{3:1 Stoichiometry}

For stoichiometry 3:1, we have N/4 positive particles and 3N/4 negative particles. In this case positive particles size is $\sqrt{3} a$, where a is the radius of negative particles. MSD calculations are shown in Figure 9. 
Figure 9 - a) MSD calculation for five different $\beta$ values (from 0 to 1.0, with variation of 0.25). Dashed line illustrates normal diffusion regime and dotted lines illustrates single-file diffusion regime. b) Insertion picture showing MSD is not equal for different values of $\beta$.

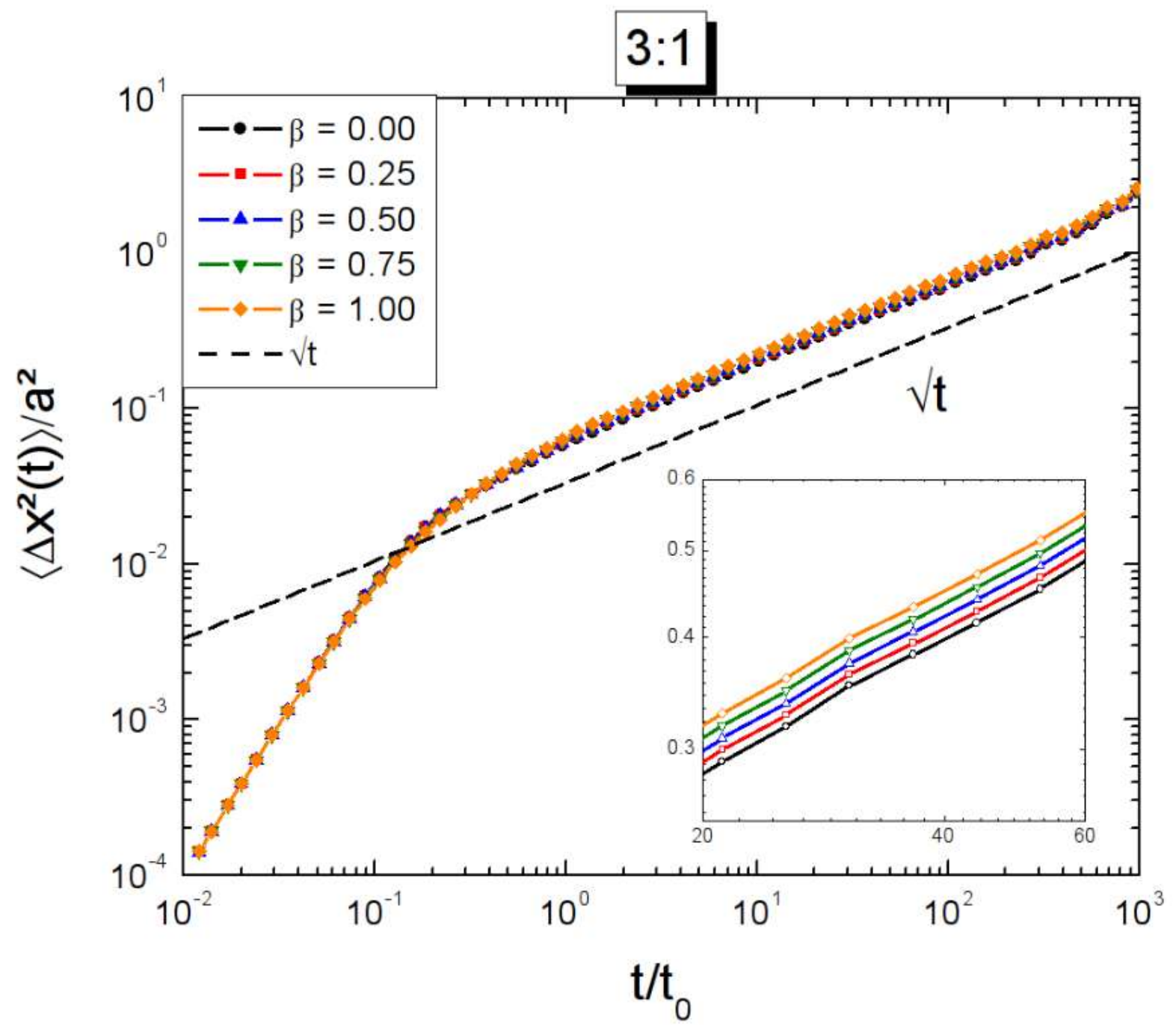

Source: Image created by the author.

For STR, the diffusive regime is still ballistic, as expected. For ITR, first of all we can observe that SFD is again obtained. To understand this result, equilibrium configurations are shown in Figure 10. 
Figure 10 - Equilibrium configuration for a system with 3:1 stoichiometry. Blue circles are positive particles and red circles are negative particles.

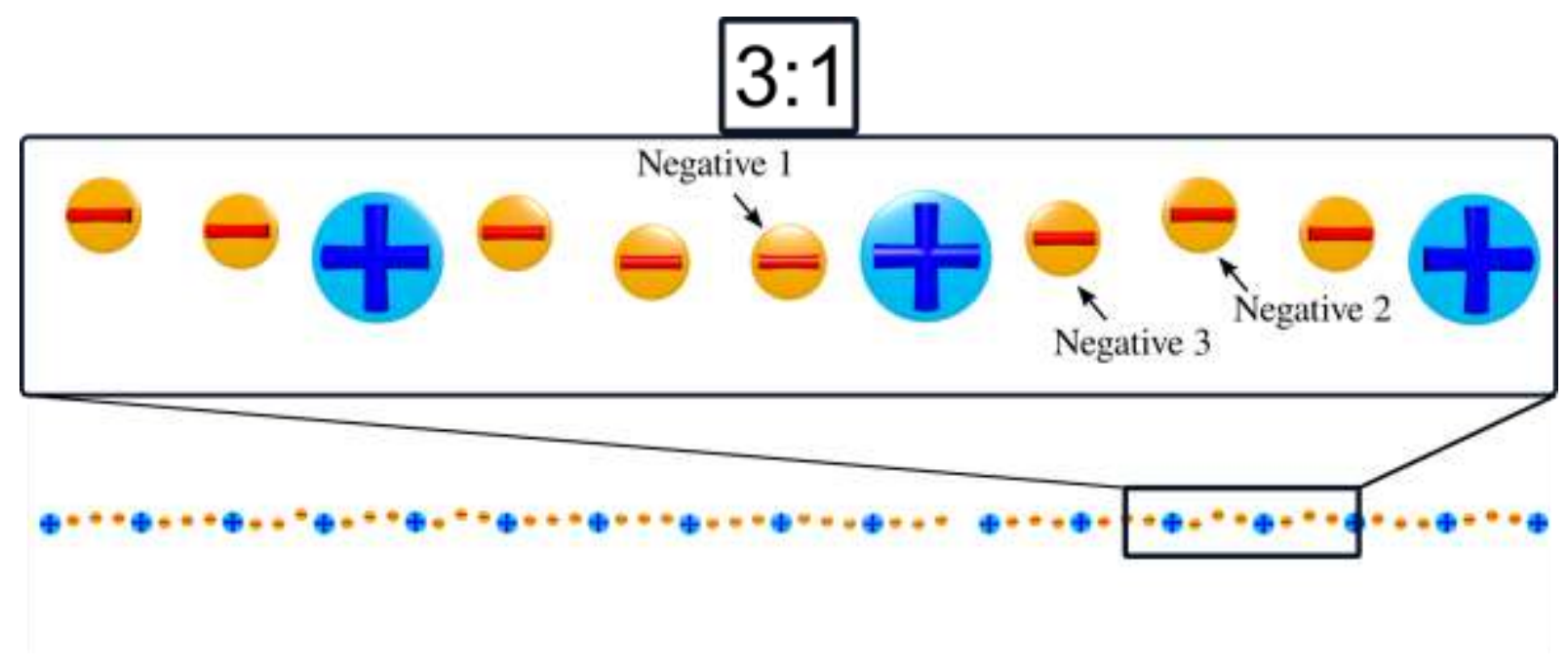

Source: Image created by the author.

When particles reach equilibrium configuration, it forms trimmer like molecules, and between them a single negative particle is free to move. The free negative particle cannot join the molecule structure because it has the same charge and then is repelled. This configuration inevitably leads to SFD because trimmers continue to act like single particles, and the interaction between trimmer-trimmer and trimmer-single particle is still only repulsive. Again we observe that when increases, clusters get lower in size, earning space to move, as in 2:1 situation. Final configuration leads us to expect that single particles diffuse in a different manner than trimmer particles. MSD for different particles are shown in Figure 11. 
Figure 11 - MSD for a) $\beta=0$ and b) $\beta=1: 0$. Black solid line is MSD curve for positive particles, red circles are MSD curve for Negative 1 particles, blue solid line is MSD curve for Negative 2 particles and green solid line is MSD curve for Negative 3 particles.

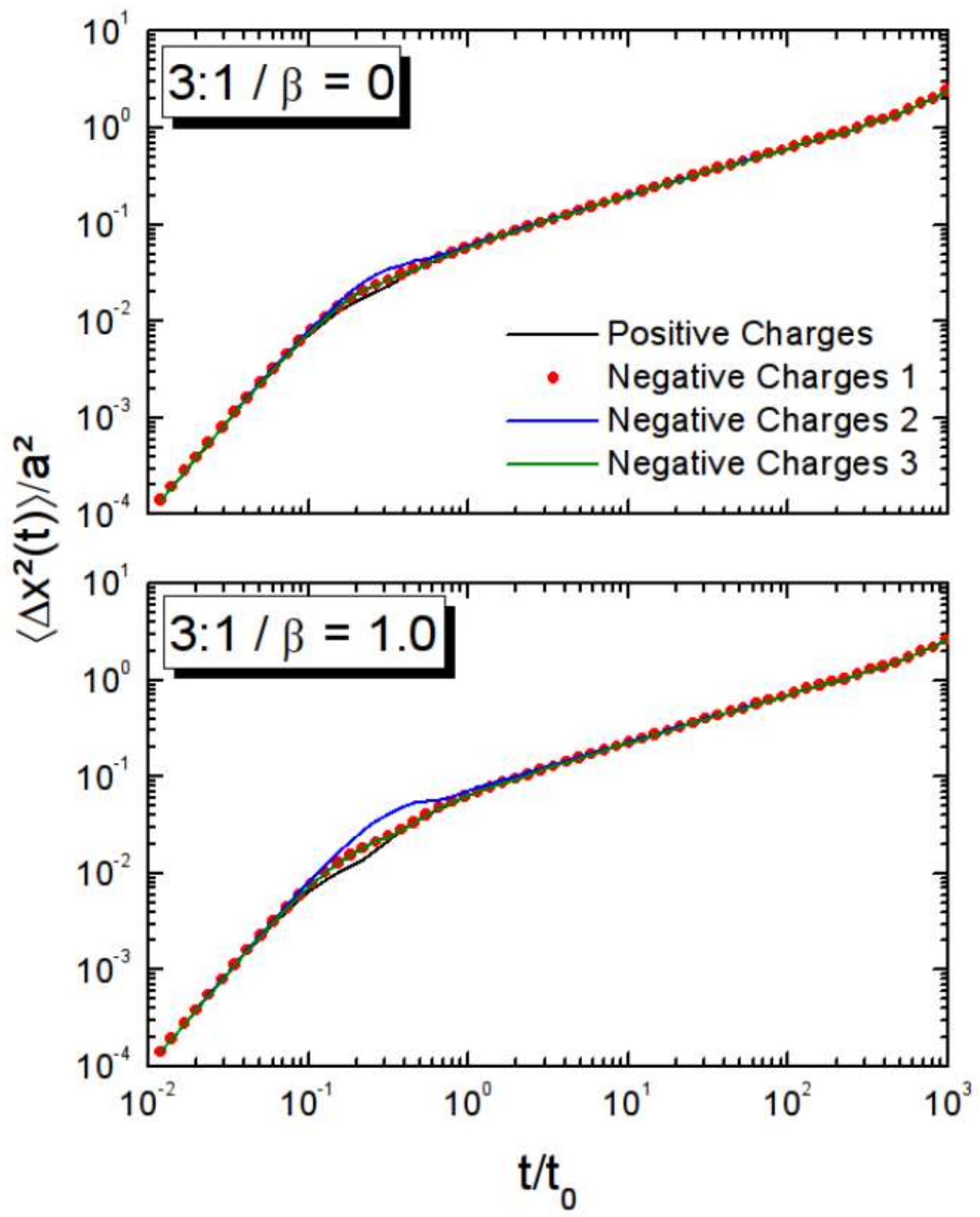

Source: Image created by the author.

We can observe two points: (i) particles Negative 2 diffuse more than the others. This can be explained by remembering that Negative 2 is the particles that are not attached to trimmer molecules, then these particles are not so in uenced by attractive forces as the others negative particles. (ii) Particles Negative 1 and Negative 3 have higher mobility than positive ones. This happens because sometimes the thermal force breaks the molecule temporarily, and then they diffuse more than positive ones that are attached to the other negative particle. Another important observation is that MSD for different charges is different for different values, as shown in Fig. 10. We can observe that for $\beta=0$, particles all diffuse in the same manner, because there is no attraction between particles, and consequently no molecule formation. When $\beta=0.50$, positive 
particles, Negative 1 and 3, and Negative 2 particles have different mobilities. When $\beta=1.0$, the difference in mobility increases also. It is important to observe that for $\beta=1.0$, we have an approximation of Negative 1 , Negative 3 and positive particle diffusion MSD curves. This happens because for higher values, trimmers are attached in a strong manner, so that they do not break so easily as for lower values, having almost equal diffusion coefficients.

\section{Conclusion}

We studied a system of oppositely charged particles, interacting through a Yukawa potential, quasi-one dimensional parabolic confinement. For three different stoichiometries and different attraction intensities, the MSD analysis showed that for the case of stoichiometry $1: 1$, when the attraction between particles becomes sufficiently strong $(\beta>0.75-1.0)$, the system starts to agglomerate, making possible the existence of diffusive regimes between SFD and normal diffusion, without changing confinement strength. This happens because attractive interactions between neighbors are stronger than repulsive interactions so that particles move faster as they approach their neighbors. It was also observed that for $\beta=0.75$ temperature breaks particle aggregation so that they diffuse more than for $\beta=1.0$. An interesting point is that with increasing attractive interaction, time transition between STR and ITR regimes decreases, because the agglomeration of particles gets faster and also collective motion. For 2:1 stoichiometry, SFD is always observed, no matter the value. Even with $\beta=1.0$, attractive interaction induces particles to form trimmer-like structures which behave as a screened single particle. It was also observed that in the transition from STR to ITR positive particles have a lower mobility, expected because positive particles have bigger size. Because positive charge is bigger than negative charge, this implies that negative particles go to positive particle direction, which leads to lower diffusion coefficient by positive particles in STR-ITR transition. For 3:1 stoichiometry, SFD is always observed for different attraction intensities. In this case, trimmers have a negative particle between, but repulsive interaction is still stronger than attractive one. In 3:1 situation, negative particles between trimmers diffuse more than the others. It is possible to observe that negative trimmers particles have higher mobility than positive ones. Another important observation is that MSD for different charges is different for different values.

The future perspectives of this work is to understand the effects of particles with different sizes and masses in diffusion exponent, where we expect to find subdiffusive regimes.

\section{References}

Carvalho, J. C. N., Ferreira, W. P., Farias G. A., \& Peeters, F. M. (2011). Yukawa particles confined in a channel and subject to a periodic potential: Ground state and normal modes. Phys. Rev. B 83(9), 094109. https://doi.org/10.1103/PhysRevB.83.094109

Carvalho, J. C. N., Nelissen, K., Ferreira, W. P., Farias G. A., \& Peeters, F. M. (2012). Diffusion in a quasi-one-dimensional system on a periodic substrate. Phys. Rev. E 85(2), 021136. https://doi.org/10.1103/PhysRevE.85.021136

Coupier, G., Jean, M. S., \& Guthmann, C. (2006). Single file diffusion in macroscopic Wigner rings. Phys. Rev. E 73(3), 031112. https://doi.org/10.1103/PhysRevE.73.031112

Delfau, J.-B., Coste, C., \& Saint Jean, M. (2011). Single-file diffusion of particles with long-range interactions: Damping and finite-size effects. Phys. Rev. E 84(1), 011101. https://doi.org/10.1103/PhysRevE.84.011101

Doyle, D. A., Cabral, J. M., Pfuetzner, A. K., Gulbis, J. M., Cohen, S. L, Chait, B. T., \& MacKinnon, R. (1998). The Structure of the Potassium Channel: Molecular Basis of K+ Conduction and Selectivity. Science 280(5360), 69-77. https://doi.org/10.1126/science.280.5360.69

Ferreira, W. P., Carvalho, J. C. N., Oliveira, P. W. S., Farias G. A., \& Peeters, F. M. (2008). Structural and dynamical properties of a quasi-one-dimensional classical binary system. Phys. Rev. B 77(1), 014112. https://doi.org/10.1103/PhysRevB.77.014112

Frenkel, D., \& Smit, B. (2002). Understanding Molecular Simulation: from algorithms to application: Amsterdam: Academic Press. https://doi.org/10.1016/B978-0-12-267351-1.X5000-7.

Galvan-Moya, J. E., Lucena, D., Ferreira, W. P., \& Peeters, F. M. (2014). Magnetic particles confined in a modulated channel: Structural transitions tunable by tilting a magnetic field. Phys. Rev. E 89(3), 032309. https://doi.org/10.1103/PhysRevE.89.032309 
Research, Society and Development, v. 10, n. 12, e403101220595, 2021

(CC BY 4.0) | ISSN 2525-3409 | DOI: http://dx.doi.org/10.33448/rsd-v10i12.20595

Gillespie, D. T. (1996). Exact numerical simulation of the Ornstein-Uhlenbeck process and its integral. Phys. Rev. E 54(2), 2084. https://doi.org/10.1103/PhysRevE.54.2084

Gillespie, D. T. (1996). The mathematics of Brownian motion and Johnson noise. Am. J. Phys. 64(3), 225. https://doi.org/10.1119/1.18210

Harris, E. T. (1965). Diffusion with “collisions” between particles. J. Appl. Probab 2(2), 323-338. https://doi.org/10.2307/3212197

Hernandez, J. A., \& Fischbarg, J. (1992). Kinetic analysis of water transport through a single-file pore. J. Gen. Physiol. 99(4), 645-662. https://doi.org/10.1085/jgp.99.4.645

Kollmann, M. (2003). Single-file Diffusion of Atomic and Colloidal Systems: Asymptotic Laws. Phys. Rev. Letters 90(18), 180602. https://doi.org/10.1103/PhysRevLett.90.180602

Konig, H., Hund, R., Zahn, K., \& Maret, G. (2005). Experimental realization of a model glass former in 2D. Eur. Phys. J. E 18, 287-293. https://doi.org/10.1140/epje/e2005-00034-9

Leunissen, M. E., Christova, C. G., Hynninen, A. P., Royall, C. P., Campbell, A. I., Imhof, A., Dijkstra, M., van Roji, R., \& van Blaaderen, A. (2005). Ionic colloidal crystals of oppositely charged particles. Nature (London) 437, 235-240. https://doi.org/10.1038/nature03946

Lucena, D., Galvan-Moya, J. E., Ferreira, W. P., \& Peeters, F. M. (2014). Single-file and normal diffusion of magnetic colloids in modulated channels. Phys. Rev. E 89(3), 032306. https://doi.org/10.1103/PhysRevE.89.032306

Lucena, D., Tkachenko, D. V., Nelissen, K., Misko, V. R., Ferreira, W. P., Farias, G. A., \& Peeters, F. M. (2012). Transition from single-file to twodimensional diffusion of interacting particles in a quasi-one-dimensional channel. Phys. Rev. E 85(3), 031147. https://doi.org/10.1103/PhysRevE.85.031147

Meier M. W., \& Olsen, H. D. (1989). Atlas of Zeolite framework types (second revised edition). Structure Commission of the international Zeolite Association 35(5), 875-875. https://doi.org/10.1002/aic.690350523

Morais-Cabral, J. H., Zhou, Y., \& MacKinnon, R. (2001). Energetic optimization of ion conduction rate by the K+ selectivity filter. Nature 414, 37-42. https://doi.org/10.1038/35102000

Nelissen, K., Misko, V. R., \& Peeters, F. M. (2007). Single-file diffusion of interacting particles in a one-dimensional channel. Europhys. Lett. 80(5), 56004. https://doi.org/10.1209/0295-5075/80/56004

Shevchenko, E. V., Talapin, D. V., Kotov, N. A., O'Brien, S., \& Murray, C. B. (2006). Structural diversity in binary nanoparticle superlattices. Nature (London) 439, 55-59. https://doi.org/10.1038/nature04414

Wei, Q.-H., Bechinger, C., \& Leiderer, P. (2000). Single-File Diffusion of Colloids in One-Dimensional Channels. Science 287(5453), 625-627. $10.1126 /$ science.287.5453.625 